\title{
Intake Ground Vortex Prediction Methods
}

\author{
J. P. Murphy* and D. G. MacManus ${ }^{+}$ \\ *Student and ${ }^{+}$Lecturer (corresponding author), Dept. of Aerospace Sciences, School of Engineering, Cranfield \\ University, Bedford MK43 0AL, UK, Email: d.g.macmanus@cranfield.ac.uk \\ Tel: +44 (0)1234 754735, Fax: +44 (0)1234 758207
}

\begin{abstract}
For an aircraft turbofan engine in ground operations or during the take-off run a ground vortex can occur which is ingested and could potentially adversely affect the engine performance and operation. The vortex characteristics depend on the ground clearance, intake flow capture ratio and the relative wind vector. It is a complex flow for which there is currently very little appropriate quantitative preliminary design information. These aspects are addressed in this work where a range of models are developed to provide a method for estimating the key metrics such as the formation boundary and the ground vortex size and strength. Three techniques are presented which utilize empirical, analytical and semi-empirical approaches. The empirical methods are primarily based on a large dataset of model-scale experiments which quantitatively measured the ground vortex characteristics for a wide range of configurations. These include the effects of intake ground clearance, approaching boundary layer thickness, intake Mach number and capture velocity ratio. Overall the models are able to predict some of the key measured behaviours such as the velocity ratio for maximum vortex strength. With increasing empiricism for key sub-elements of the model construction, an increasing level of agreement is found with the experimental results. Overall the three techniques provide a relatively quick and easy method in establishing the important vortex characteristics for a given headwind configuration which is of significant use from a practical engineering perspective.
\end{abstract}




\section{Accepted manuscript}

\section{Keywords}

Ground vortex, Intake, Vortex Strength, Modelling, Headwind, Particle Image Velocimetry (PIV)

\section{Nomenclature}

\begin{tabular}{|c|c|c|c|}
\hline$A_{\infty}$ & $\begin{array}{l}\text { Sucked streamtube area upstream of } \\
\text { intake, } m^{2}\end{array}$ & $V_{r}$ & Radial velocity, $m s^{-1}$ \\
\hline$D_{i}$ & Intake throat diameter, $m$ & $x, y, z$ & Cartesian coordinates, $m$ \\
\hline$D_{l}$ & Intake highlight diameter, $m$ & $\alpha$ & Empirical constant \\
\hline$h$ & $\begin{array}{l}\text { Vertical distance from lowest point } \\
\text { of intake highlight plane to the } \\
\text { ground, } m\end{array}$ & $\beta$ & Empirical constant \\
\hline$h_{L}$ & $\begin{array}{l}\text { Measurement plane height from } \\
\text { ground plane, } m\end{array}$ & $\delta$ & Boundary layer thickness, $m$ \\
\hline$H$ & $\begin{array}{l}\text { Intake centreline height from the } \\
\text { ground plane, } m\end{array}$ & $\delta^{*}$ & $\begin{array}{l}\text { Approaching boundary } \\
\text { displacement thickness, } m\end{array}$ \\
\hline$k$ & Empirical constant & $\rho^{*}$ & Density ratio $\left(=\rho_{i} / \rho_{\infty}\right)$ \\
\hline$L_{q}$ & $\begin{array}{l}\text { Height of sucked streamtube from } \\
\text { ground, } m\end{array}$ & $V_{\theta}$ & Swirl velocity, $m s^{-1}$ \\
\hline$M_{i}$ & Intake throat Mach number & $\omega$ & Vorticity, $s^{-1}$ \\
\hline$n$ & Vatistas vortex model exponent & $\sigma$ & Empirical constant \\
\hline$n_{b l}$ & $\begin{array}{l}\text { Boundary layer power law } \\
\text { coefficient }\end{array}$ & & \\
\hline$r$ & $\begin{array}{l}\text { Radial distance from centre of } \\
\text { vortex, } m\end{array}$ & $\Gamma^{*}$ & $\begin{array}{l}\text { Average total non-dimensional vortex } \\
\text { strength }\left(=\Gamma / D_{l} U_{i}\right)\end{array}$ \\
\hline$r_{c}$ & Vortex core radius, $\mathrm{m}$ & $\Gamma$ & Average vortex circulation, $m^{2} s^{-1}$ \\
\hline$r^{*}$ & $\begin{array}{l}\text { Non-dimensional radial distance } \\
\left(=r / r_{c}\right)\end{array}$ & $\Gamma_{\infty}$ & Approaching circulation, $m^{2} s^{-1}$ \\
\hline$u, v, w$ & Cartesian velocity components, $m s^{-1}$ & $\Gamma_{i d}$ & Induced circulation, $m^{2} s^{-1}$ \\
\hline$U_{\infty}$ & Tunnel free-stream velocity, $m s^{-1}$ & $\Psi$ & Intake yaw angle, $d e g$ \\
\hline$U_{i}$ & Average intake throat velocity, $m s^{-1}$ & $\Pi_{1}$ & Empirical constant \\
\hline$U^{*}$ & Velocity ratio $\left(=U_{i} / U_{\infty}\right)$ & $\Pi_{2}$ & Empirical constant \\
\hline$U^{* *}$ & $\begin{array}{l}\text { Velocity ratio based on average } \\
\text { approaching velocity within sucked } \\
\text { streamtube }\left(=\mathrm{U}_{\mathrm{i}} / \bar{U}_{\infty}\right)\end{array}$ & & \\
\hline$U_{\text {crit }}^{*}$ & Vortex blow-away velocity ratio & & \\
\hline$U_{\max }^{*}$ & $\begin{array}{l}\text { Velocity ratio corresponding to } \\
\text { maximum vortex strength }\end{array}$ & & \\
\hline$U_{d}^{*}$ & $\begin{array}{l}\text { Critical velocity ratio parameter } \\
\text { (Model 3) }\end{array}$ & & \\
\hline$U_{R}^{*}$ & $U_{c r i t}^{*} / U_{\max }^{*}$ & & \\
\hline$U_{\text {trans }}^{*}$ & $\begin{array}{l}\text { Transition boundary velocity ratio } \\
\text { (Model 3) }\end{array}$ & & \\
\hline
\end{tabular}




\section{Introduction}

Ground vortex formation is a potential flow feature that occurs when jet engines are operated in static or near static conditions close to a ground plane. Under these conditions, at least one vortex extends from the ground and is sucked into the engine. The ingested vortex or vortices are potentially detrimental to engine operation and can ultimately lead to foreign object damage [1-2], mass flow and total pressure distortion [3] as well as fan vibration issues [4]. Previous research has identified two fundamentally different ground vortex formation mechanisms. The first is the headwind mechanism in which vortex formation occurs due to the concentration, stretching and intensification of ambient vortex lines associated with the dominant vorticity source $[5,6]$. Typically the vorticity within the approaching boundary layer is the dominant aspect and leads to the formation of two counter rotating vortices. The second mechanism, however, relates to an intake in a significant crosswind condition (yaw angles greater than $45^{\circ}$ ) in which the ground vortex is primarily generated from vorticity associated with the intake external surface [5]. Only a single vortex is generated in crosswind but it is significantly stronger than the vortices created under headwind conditions [7].

To determine the impact of the vortex on the downstream turbo-machinery it is essential to have knowledge of its strength, size, ingestion location and pressure loss characteristics. Previous experimental work [7-8] has quantitatively shown that the above characteristics strongly depend on a number of geometric and aerodynamic parameters including the velocity ratio, $U_{i} / U_{\infty}$, the non-dimensional height of the intake, $h / D_{l}$, the approaching boundary layer thickness ratio, $\delta^{*} / D_{l}$, and the intake yaw angle, $\psi$.

During the preliminary design phase of an engine configuration it is useful to have prior knowledge of the vortex characteristics to enable an assessment of its potential impact on the aircraft engine. Currently this is achieved by conducting full-scale engine tests, model wind tunnel experiments or computational analyses which are both expensive and time consuming. The motivation for this paper is therefore to present three simple prediction tools which can be applied to headwind configurations to determine the vortex strength. All techniques provide a quick and reasonably accurate indication of the operating points that generate the strongest vortex for a given headwind configuration as well as its magnitude. The first method is derived purely from empirical correlations whereas the second is a simple analytical tool which implements a more fundamental approach. Due to the modelling simplifications, the latter tool is less accurate but is more instructive in understanding the fundamental mechanisms associated with headwind ground vortex formation. The third technique, however, uses the simple analytical model in conjunction with a core embedded level of empiricism to achieve a greater level of accuracy

The paper is structured by first describing the methodology of the model-scale experiments that have been conducted which are described in more detail in [7-10]. This is followed by a summary of the key experimental results under headwind conditions that are pertinent to the vortex strength models. The empirical model is then presented and compared with the experiments and is then followed by the analytical vortex strength prediction technique. Finally a semi-empirical model is presented and the performance of which is then also assessed against the experimental dataset for a range of configurations.

\section{Description of Experiments}

\section{A. Test facility and model}

The experiments were conducted in the Cranfield University low-speed wind tunnel which has a $2.4 m \times$ $1.8 m$ working section. A cylindrical model intake of approximately $1 / 30^{\text {th }}$ scale was used with an inside diameter of $0.1 \mathrm{~m}$. The intake Reynolds number was fixed at $1.26 \times 10^{6}$ based on the inner diameter and average intake velocity. Due to the low Reynolds number, transition strips were placed on both the inside and outside of the intake lip to promote transition and to avoid premature laminar separations. In addition, the lip geometry was modified relative to a large-scale intake and consisted of elliptical elements, with a major-to-minor axis ratio of 2. The model did not include a central hub or a rotating fan. The intake mass-flow was provided by a suction system which was connected to a $60 \mathrm{~m}^{3}$ vacuum tank. The flow was controlled using a quick release shutter valve and the required steady mass flow was achieved approximately two seconds after initiation. A run time of approximately 20 seconds was achieved at a constant maximum mass flow of $1.49 \mathrm{~kg} / \mathrm{s}\left(M_{i}=0.58\right)$. The mass 
flow was monitored for the duration of the experiments using static pressure measurements in the intake and was found to be steady throughout with a typical variation of $0.1 \%$.

\section{PIV methodology}

A TSI stereoscopic PIV system was used to acquire the three-components of velocity on a plane. The PIV system consists of two four-mega pixel cameras orientated at $\pm 45^{\circ}$ to the measurement plane with both operating in partial scatter with respect to the laser. For the static ground plane experiments, the cameras were equipped with $60 \mathrm{~mm}$ focal length lens and were positioned underneath the wind tunnel floor. For the rolling ground plane experiments, the cameras were positioned inside the tunnel working section using $105 \mathrm{~mm}$ focal length lens. A New Wave Solo 120XT Nd:Yag laser with a wave length of $532 \mathrm{~nm}$ was used and a $1.5 \mathrm{~mm}$ light sheet was generated using a combination of a spherical plano-concave and a cylindrical plano-convex lenses. The position of the light sheet relative to the intake is indicated in Fig. 1d. The resulting flow-field snapshots were acquired at a frame rate of $7.5 \mathrm{~Hz}$. The flow was seeded by a Laskin-type seeder using Di-2-EthylhexylSebacat (DEHS) oil which delivered a mean particle diameter of $1 \mu \mathrm{m}$. The seeding rake was fixed in position for all experiments and was located upstream of the working section ahead of the wind tunnel boundary layer suction slots.

\section{Analysis Methodology}

The PIV images were post-processed using TSI Insight3G software version 8.0.5. It was found that a deformation grid, along with the FFT algorithm was the best method to use for this flow-field. The method allows the procedure to be recursively implemented so that a multi-grid approach can be used to further increase the signal-to-noise ratio. A $64 \times 64$ interrogation spot was used initially with $50 \%$ overlap to maximise the correlation peak and ensure good sub-pixel accuracy in the displacement estimate for the windows off-sets. A pass validation was also implemented which included a median filter to remove spurious vectors and $3 \times 3$ smoothing to remove any small scale noise present. The final iteration used a $32 \times 32$ (with $50 \%$ overlap) spot area to improve the resolution. This resulted in a measurement resolution of $0.78 \mathrm{~mm}$.

The position of maximum vorticity was successfully used to identify the vortex centre. This parameter was preferred to more advanced identification parameters such as the swirling strength, $Q$ and the eigenvalues of the velocity gradient tensor, $\lambda_{2}$ [11] because it can discriminate between positive and negative rotating vortices. This therefore enables such vortices to be identified and their respective characteristics to be determined individually. The vortex parameters were identified using the vorticity disk method [12]. The output of this method is a circumferentially averaged swirl velocity distribution as a function of radial distance from the centre of the vortex. The method is applied by first identifying the vortex centre location and then integrating vorticity over circular areas with increasing radial distance, $r$. The swirl velocity distribution is then obtained by dividing the local circulation at each radial position by $2 \pi r$ (Eq. 1$)$.

$V_{\theta}(r)=\frac{\Gamma(r)}{2 \pi r}$

The total individual vortex strength is obtained by integrating all positive vorticity over the whole circular domain for the positive vortex and vice versa for the negative vortex. The total average non-dimensional strength of the vortex system, $\Gamma^{*}$, is determined by averaging the vortex strengths over all snapshots summing the magnitudes and normalising by the intake velocity, $U_{i}$, and highlight diameter, $D_{l}$ (Eq. 2). Further details are provided in Murphy [9].

$$
\Gamma^{*}=\frac{\left|\Gamma^{+}\right|+\left|\Gamma^{-}\right|}{U_{i} D_{l}}
$$




\section{Experiment Uncertainties}

The measurement uncertainties comprise of a combination of sources such as those due to the model positioning, measurement transducers, random errors and the data acquisition system. Following the method of Taylor [13], these elements have been assessed to provide an estimate of the overall uncertainty for each measurement type. The non-dimensional height was set with an uncertainty of $h / D_{l}=0.25 \pm 3.6 \%$. The freestream velocity ranged from 10 to $40 \mathrm{~m} / \mathrm{s}$ with a typical overall uncertainty of $\pm 1.1 \%$. The average intake velocity was measured using total and static pressure measurements within the intake duct and was typically found to be $185 \mathrm{~m} / \mathrm{s} \pm 2.4 \%$. For the duration of the run the intake velocity was reasonably constant throughout with a typical deviation of $0.15 \%$ of the average. The velocity ratio, $U_{i} / U_{\infty}$, ranged from $\infty$ to 4.6 and for a typical median velocity ratio of 6.1 the uncertainty in $U_{i} / U_{\infty}$ was $\pm 2.6 \%$. The rolling road velocity, $U_{g}$, was measured to within $0.05 \mathrm{~m} / \mathrm{s}$. Belt suction was applied throughout the testing to ensure that the rolling road did not lift or move laterally during tests.

For the PIV velocity measurements an uncertainty band was estimated following the analysis presented by Raffel et al [14] which is based on synthetic PIV images. The analysis used an FFT correlation engine and a three point Gaussian peak fit algorithm which is relevant to this research. The correlation peak was estimated to be measured to within \pm 0.06 pixels for each camera. Misalignment of the light sheet with the calibration plate is expected to be the largest source of error, with the centre of the light sheet being, at worst $\pm 0.25 \mathrm{~mm}$ off centre. Using error estimates from Petracci et al [15] this is expected to result in a maximum error of 0.13 pixels. The total error is expected to be no worse than \pm 0.15 pixels. This equates to an in plane velocity error of $1.61 \mathrm{~m} / \mathrm{s}$ or a typical error of $\pm 3.2 \%$. Since the half-angle between the cameras was at $45^{\circ}$, the out of plane velocity error is equal to the in-plane error in $\mathrm{m} / \mathrm{s}$ [16]. The vorticity error is estimated to be approximately $\pm 1875 / \mathrm{s}$ based on the maximum velocity error. This leads to a worst case error in the circulation of approximately $\pm 0.3 \mathrm{~m}^{2} / \mathrm{s}$. Further details on uncertainty analysis and also the experiment apparatus and procedures are presented in Murphy [9].

\section{Flowfield Quantification}

Under quiescent conditions $\left(U_{i} / U_{\infty}=\infty\right)$ a pair of contra-rotating vortices form ahead of the intake highlight plane as illustrated in Fig. 1a which shows an example snapshot of the in-plane velocity vectors. At this velocity ratio $\left(U_{i} / U_{\infty}=\infty\right)$, the suction induced flow which is drawn from the region between the intake and the ground dominates. Consequently, the vorticity associated with this flow results in a contra-rotating vortex topology where the right hand vortex has a clockwise orientation (Fig. 1b). As a headwind component is added and the velocity ratio reduces, the flow mode changes and the pair of contra-rotating vortices switch their sense of rotation (Fig. 1c). This is as a result of the addition of the approaching boundary layer vorticity which now dominates as illustrated in Fig. 1d. Under quiescent conditions, when the intake ground clearance increases, the induced velocities immediately adjacent to the ground plane reduce. This leads to lower vortex strength due to the lower vorticity magnitude being generated at the ground plane. This is shown in Fig. 2, where the nondimensional vortex strength is plotted against non-dimensional height. Fig. 2 also shows that $\Gamma^{*}\left(=\Gamma / U_{i} D_{l}\right)$ is independent of intake Mach number, $M_{i}$, and therefore scales with the intake velocity, $U_{i}$, for these cases using a fixed geometry. 
(a)

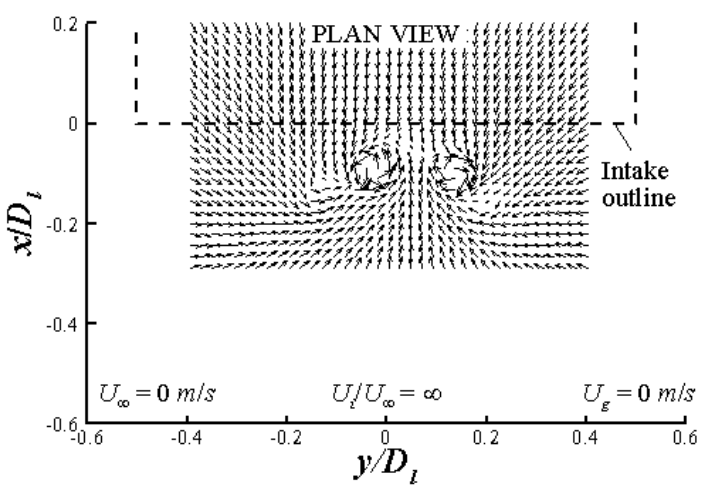

(c)

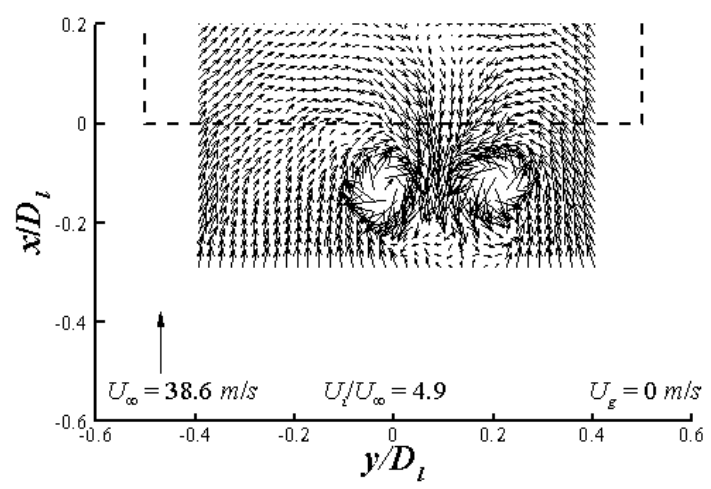

(b)

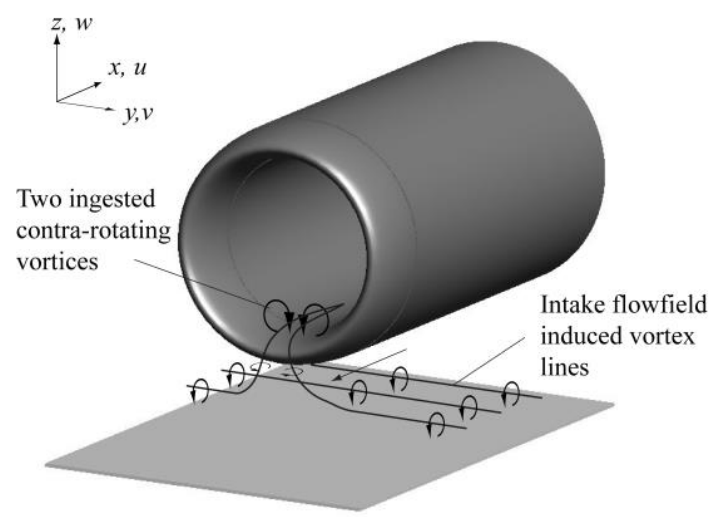

(d)

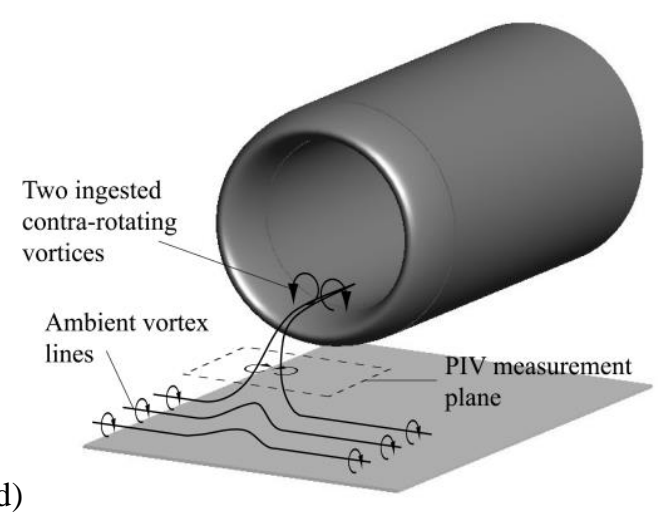

Fig. 1 Ground vortex formation under (a) quiescent conditions and (c) headwind conditions showing the in-plane $u$-v velocity vectors at the PIV measurement plane (only every $3^{\text {rd }}$ vector shown) with (b) and (d) showing the respective deformation and ingestion of the dominant vortex lines.

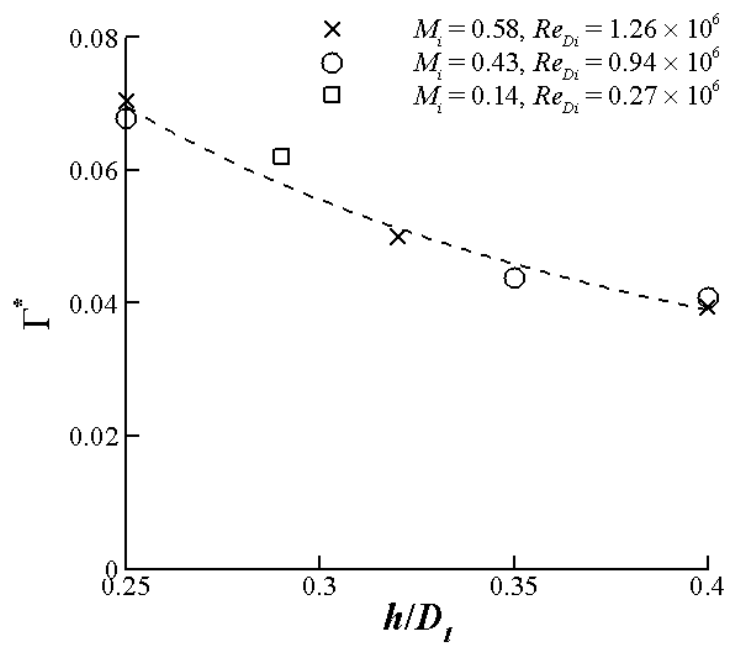

Fig. 2 Non-dimensional vortex strength, $\Gamma^{*}$, against intake ground clearance, $h / D_{l}$, for various different intake Mach numbers $\left(U_{i} / U_{\infty}=\infty\right)$

With the introduction of a headwind velocity an additional source of vorticity is introduced into the flowfield which is associated with the approaching boundary layer. There are now two primary vorticity sources for ground vortex formation which are related with the approaching and induced flowfields. The convection of the vortex lines associated with the approaching flowfield is shown in Fig. 1d. At high headwind speeds and low velocity ratios $\left(U_{i} / U_{\infty}>20\right)$, this flowfield topology dominates and two counter rotating vortices are ingested into the intake, as indicated in Fig. 1d. Since the dominant vorticity source approaches the intake from the 
opposite direction, the vortices have a reversed sense of rotation in comparison to quiescent conditions (Fig. 1c). However, for low non-zero headwind speeds in which the velocity ratio is sufficiently high $\left(U_{i} / U_{\infty}>20\right.$ for $h / D_{l}$ $=0.25$ ), the induced vorticity dominates and the vortices rotate in accord with Fig. 1a at the PIV measurement plane. This has also been quantitatively verified by Brix et al [17].

Fig. 3 shows the total non-dimensional vortex strength against velocity ratio under headwind conditions for three non-dimensional heights. The quiescent configuration, for each $h / D_{l}$, is also included in the figure and is indicated by the $U^{*}=\infty$ data point. At the lowest ground clearance of $0.25\left(h / D_{l}\right)$, the introduction of a modest headwind velocity $\left(U_{\infty}=9.8 \mathrm{~m} / \mathrm{s}, U_{i} / U_{\infty}=19.8\right)$ leads to no notable change in the overall vortex strength (Fig. 3). However, as the headwind velocity is increased, and the velocity ratio is reduced below 12 $\left(U_{i} / U_{\infty}\right)$, the vortex strength sharply increases and eventually reaches a local maximum. With further reductions in the velocity ratio a sharp reduction in vortex strength is observed. Due to tunnel limitations the velocity ratio could not be reduced below 4.6.

The general trend of vortex strength with velocity ratio for the other investigated non-dimensional heights is comparable, although there are differences. As the ground clearance is increased to $0.32\left(h / D_{l}\right)$ the peak vortex strength reduces and there is an increase in the corresponding velocity ratio of the local maximum $\left(U_{\max }^{*}\right)$. As with the lowest non-dimensional height of $0.25\left(h / D_{l}\right)$, when the velocity ratio is reduced below the condition of maximum strength $\left(U_{\max }^{*}\right)$, the vortex strength rapidly diminishes until a vortex no longer forms.

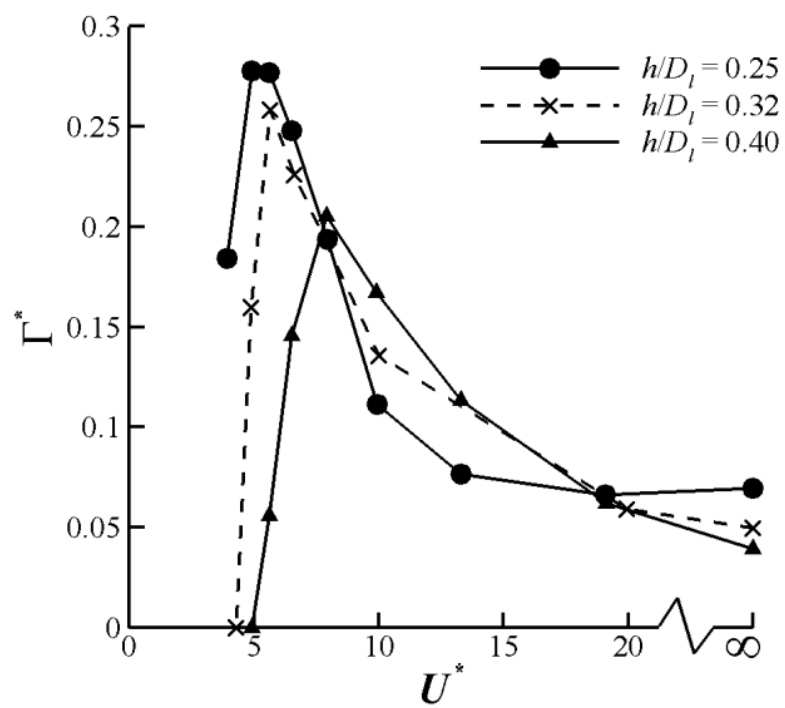

Fig. 3: Effect of velocity ratio, $U^{*}$, on the non-dimensional vortex strength, $\Gamma^{*}$, for various ground clearances $\left(\delta / D_{l}=0.11, M_{i}=\mathbf{0 . 5 8}\right)$.

The variation in the total vortex circulation with velocity ratio is a result of two opposing mechanisms. As the approaching headwind velocity increases the approaching vorticity magnitude for the vortex increases. However, in tandem, the sucked streamtube size also reduces. Since the total vortex strength is dependent on the integrated vorticity within the sucked streamtube, the balance between these two mechanisms leads to a local peak in the vortex strength. When the sucked streamtube size reduces sufficiently such that no interaction occurs with the ground plane a ground vortex can no longer form and its strength reduces to zero. The velocity ratio at which this occurs is denoted by $U_{c r i t}^{*}$. Similar observations are also observed in terms of the distortion coefficient variation with velocity ratio within the intake duct as reported in [8-9]. 


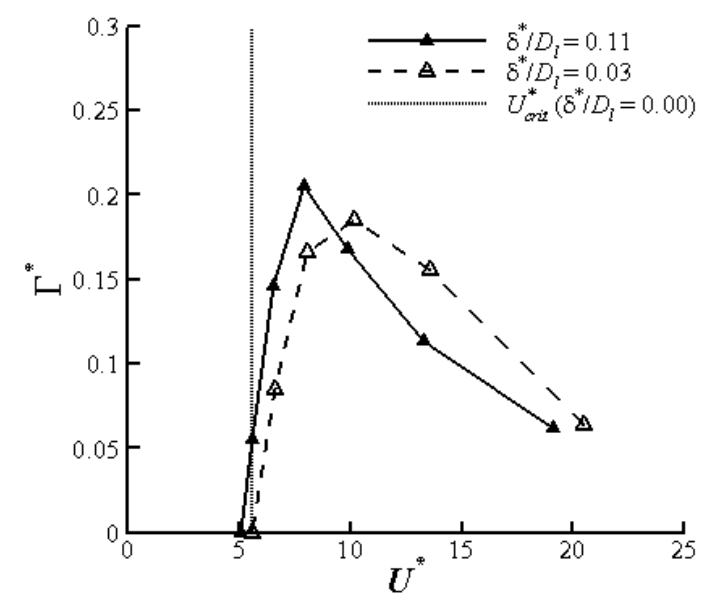

Fig. 4: Effect of boundary layer thickness on the vortex strength as a function of velocity ratio $\left(h / D_{l}=0.4, M_{i}=0.58\right)$.

The influence of the approaching boundary layer thickness, $\delta^{*} / D_{l}$, has also been examined on the ground vortex characteristics. It is important to establish the sensitivity of this parameter as in practise the intake is immersed in an atmospheric boundary layer, which is at least an order of magnitude larger than the intake dimension. The experimental observations reveal an important finding with regards to the vortex blow-away condition, $U_{c r i t}^{*}$. Fig. 4 plots the vortex circulation variation with velocity ratio for a $\delta^{*} / D_{l}$ equal to 0.11 and 0.03 , at a ground clearance of $0.4\left(h / D_{l}\right)$. Also included in the figure is the predicted velocity ratio at which the sucked streamtube lifts off the ground plane which for an $h / D_{l}=0.4$ is denoted by $U_{\text {crit }}^{*}$ (Fig. 4). This has been predicted from continuity considerations assuming a circular axi-symmetric sucked streamtube with a uniform flow (i.e. $\delta^{*} / D_{l}=0$ ) and is calculated using Eq. (3). Although previous research has looked at mass conversation to predict the vortex blow-away condition [18], this is the first study to experimentally verify that the critical velocity ratio occurs when the sucked stream-tube is calculated to lift of the ground plane.

$U_{c r i t}^{*}=\left(\frac{U_{i}}{U_{\infty}}\right)_{c r i t}=4 \rho^{*}\left(\frac{D_{l}}{D_{i}} \frac{h}{D_{l}}+\frac{D_{l}}{2 D_{i}}\right)$

As with the effect of ground clearance the general variation of vortex strength with velocity ratio for different boundary layer configurations is the same although there are differences. With $\delta^{*} / D_{l}$ reducing from 0.11 to 0.03 the peak vortex strength moderately reduces from 0.21 to 0.19 with the corresponding velocity ratio increasing from 7.9 to 10.2 (Fig. 4). In addition, the velocity ratio at which the vortex no longer forms also increases from 5.1 to $5.6\left(U^{*}\right)$.

These observations were anticipated and are due to an alteration in the sucked streamtube interaction with the ground plane, for a given velocity ratio, between the two approaching boundary layer configurations. For the smaller approaching boundary layer $\left(\delta^{*} / D_{l}=0.03\right)$ the velocity profile will be more uniform and therefore the average velocity within the sucked streamtube will be higher in comparison to the $\delta^{*} / D_{l}=0.11$ case. From mass conservation the area of the sucked streamtube upstream of the intake is dependent on the ratio of the area weighted average intake velocity to the area weighted average free-stream velocity within the sucked streamtube. Hence for a given velocity ratio, $U^{*}$, reducing the approaching boundary layer thickness will reduce the sucked streamtube size and therefore its consequent interaction with the ground plane. As a result the vortex strength characteristic therefore appears to have shifted to higher velocity ratios relative to the $\delta^{*} / D_{l}=0.11$ configuration (Fig. 4). Since $U_{\text {crit }}^{*}$ in Fig. 4 has been computed for an infinitely small approaching boundary layer $\left(\delta^{*} / D_{l}=0.00\right)$ the predicted vortex blow-away condition agrees well with the $\delta^{*} / D_{l}=0.03$ dataset (Fig. 4). This confirms that the vortex blow-away condition is met when the sucked streamtube lifts off the ground plane and will be used in the vortex strength prediction tools outlined below. In terms of the peak vortex strength, as noted in [9], the integrated vorticity across the height of the approaching boundary layer is largely the same for 
the two boundary layer configurations. This explains why the maximum vortex strength is reasonably similar between the two cases. Similar observations are also seen for the lower ground clearance $\left(h / D_{l}=0.25\right)$ and as well as for the in-duct total pressure distortion survey [8].

\section{A. Aerodynamic Self-Similarity}

When the distributions of non-dimensional vortex strength against velocity ratio, such as those shown in Fig. 3 and Fig. 4, are normalised by the appropriate local maximum values and the corresponding velocity ratio, $\left(U_{i} / U_{\infty}\right)_{\max }$, the profiles exhibit a self-similar trend (Fig. 5). This data reduction is based on a broad range of configurations including three non-dimensional heights and three approaching boundary layer thicknesses. The trend exhibits a power law distribution with reducing velocity ratio until the local peak is reached. Further reductions in velocity ratio, where the vortex strength reduces, can be represented using a linear approximation. The intersection of this linear line with the $x$-axis gives the velocity ratio $\left(U_{c r i i}^{*} / U_{\max }^{*}\right)$ at which the vortex is blown away. The PIV vortex strength measurements indicate that the vortex blow-away velocity ratio $\left(U_{c r i t}^{*}\right)$ is approximately $65 \%$ of the velocity ratio at which the maximum vortex strength occurs $\left(U^{*}{ }_{\max }\right)$. This ratio will be subsequently used in the empirical vortex strength prediction tool presented below.

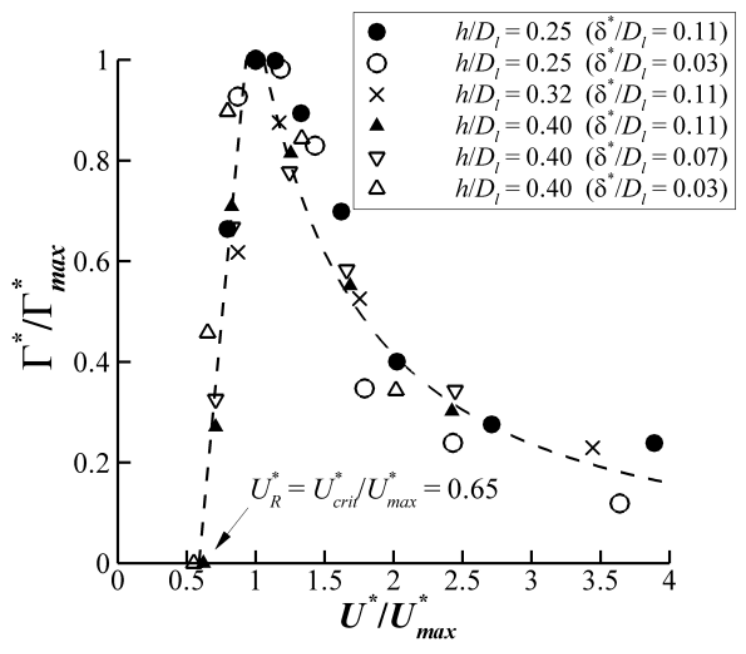

Fig. 5 Normalised vortex strength against normalised velocity ratio for a range of headwind configurations $\left(M_{i}=\mathbf{0 . 5 8}\right)$.

\section{B. Rolling Road Experiments}

As mentioned above there are two primary vorticity sources for headwind ground vortex formation. These are associated with the approaching and induced flowfields. For a headwind static ground configuration both sources are present and it is difficult to establish the dominance and interdependence of both across the range of operating velocity ratios. One method of removing the approaching vorticity source, and therefore isolating the induced circulation, is to employ the moving ground technique. The ground velocity, denoted by $U_{g}$, is set equal to the tunnel wind speed, $U_{\infty}$ (Fig. 6b) such that $\Delta U$ is equal to zero (Eq. (4). This is performed in conjunction with an upstream suction system which removes the approaching boundary layer. A range of increasing headwind speeds was examined to establish the effect of velocity ratio without an approaching boundary layer. At each investigated velocity ratio the difference between the ground velocity, $U_{g}$, and the freestream velocity, $U_{\infty}$, denoted by $\Delta U$ (Eq. (4) is set at zero. Other non-zero magnitudes of $\Delta U$ have also been examined, but are not discussed in depth within this paper (refer to [10] for further details). As opposed to a static ground plane case where a boundary layer profile approaches the intake (Fig. 6a), for this configuration $(\Delta \mathrm{U}=0 \mathrm{~m} / \mathrm{s})$, the velocity profile is nearly uniform upstream of the intake (Fig. 6b) and therefore almost no approaching vorticity source is present. Full details of the experiment set-up and influence of the moving ground on the ground vortex flowfield and vortex characteristics can be found in [9]. In this paper, however, the focus is purely on the vortex strength variation - the results of which are used in the semi-empirical vortex strength model presented below. 
$\Delta U=U_{\infty}-U_{g}$

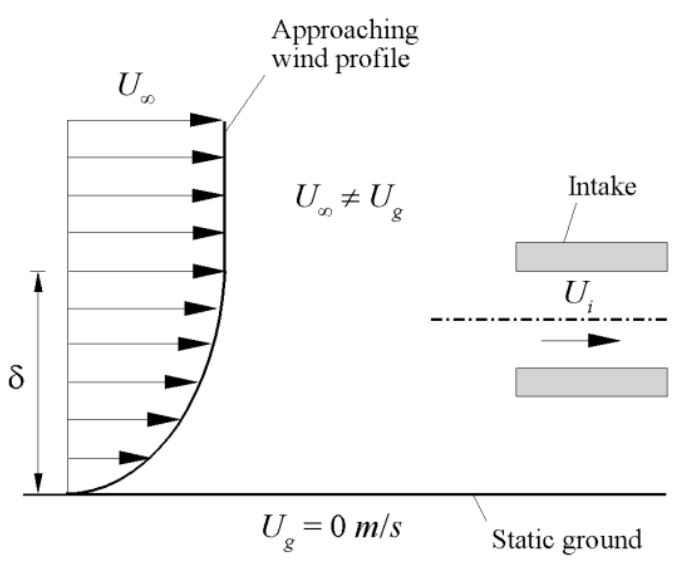

(a)

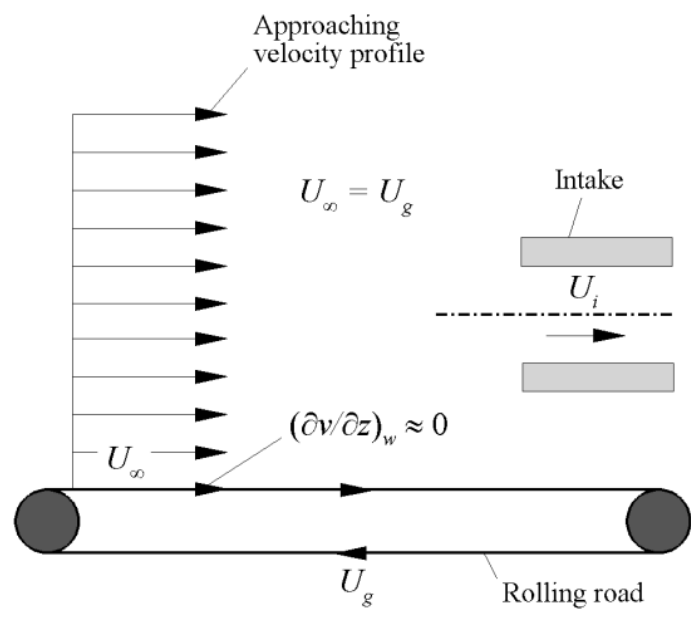

(b)

Fig. 6: Schematic of (a) the static and (b) the synchronised rolling road configurations.

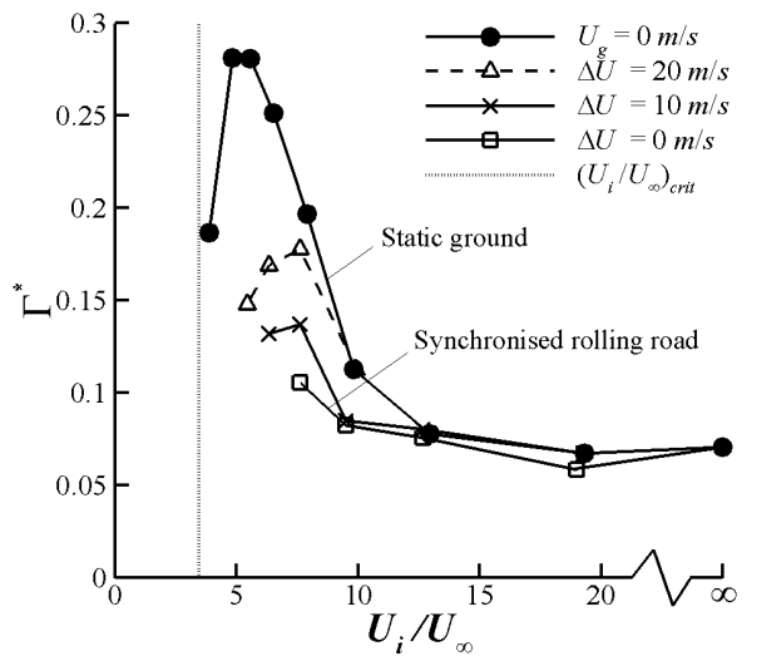

Fig. 7: Vortex strength against velocity ratio comparing the static and moving ground cases $\left(h / D_{l}=0.25\right.$,

$$
\left.M_{i}=\mathbf{0 . 5 8}\right)
$$

The effect of increasing the ground speed $\left(U_{g}\right)$ synchronized with the tunnel free-stream velocity $\left(U_{\infty}\right)$, (such that the difference in magnitude between the two velocities is zero) on the total average non-dimensional vortex strength is presented in Fig. 7. The static ground configurations $\left(U_{g}=0 \mathrm{~m} / \mathrm{s}\right)$ for an increasing headwind as well as various magnitudes of $\Delta U$ are also included in the figure for comparison. For low ground speeds $\left(U_{g}\right.$ $=0-15 \mathrm{~m} / \mathrm{s})$, and correspondingly high velocity ratios $\left(U_{i} / U_{\infty}=12.7-\infty\right)$, the total vortex strength does not depend on the rolling road and is similar to the corresponding static ground configurations (Fig. 7). Since there is very little approaching boundary layer vorticity flux, this indicates that the dominant vorticity source, for a static ground $\left(U_{g}=0 \mathrm{~m} / \mathrm{s}\right)$, at intermediate to high velocity ratios, is the suction induced vorticity. As $U_{g}$ increases further and velocity ratio $\left(U_{i} / U_{\infty}\right)$ reduces, a deviation in the strength from the static road configurations is seen when the velocity ratio reaches approximately 9.5 (Fig. 7). At this headwind speed, for the static ground plane, the approaching boundary layer vorticity becomes influential on the total vortex strength. With a further increase in the ground speed, $U_{g}$, to $25 \mathrm{~m} / \mathrm{s}\left(U_{i} / U_{\infty}=7.5\right)$ there is approximately a $50 \%$ 
reduction in the strength in comparison to the static ground case at the same velocity ratio (Fig. 7). As the ground speed increases further and the velocity ratio, $U_{i} / U_{\infty}$, reduces to 6.2 , no vortex was detected in the measurement area and therefore no additional measurements could be taken. Overall this illustrates that there is a large reduction in the ground vortex strength when comparing a static and a moving ground configuration. The additional rolling road configurations shown in Fig. 7 in which $\Delta U$ is non-zero (i.e. $\Delta U=10$ and $20 \mathrm{~m} / \mathrm{s}$ ) concur with the synchronised rolling ground plane results $(\Delta U=0 \mathrm{~m} / \mathrm{s})$. Since these configurations lie somewhere in between the static ground and synchronised rolling road tests it is not surprising that the strength of the vortex also occurs somewhere in-between.

It should be noted that the effect of the intake Reynolds number is expected to have a modest impact on the vortex strength. In a parallel study to this research, CFD predictions were conducted by Zantopp [19] for the same intake model and configurations. Within Zantopp's work, full scale calculations were performed under headwind conditions and the results confirmed that the vortex strength scales with the intake velocity, $U_{\infty}$, and intake diameter, $D_{l}$ for a given $\delta^{*} / D_{l}$ ratio . This was also found to be the case under cross-wind conditions [20]. Although the ground vortex strength is not sensitive to the intake Reynolds number, the $D C_{60}$ is expected to depend on it as it will affect the impact of the ingested vortex on the intake boundary layer.

\section{Empirical Vortex Strength Prediction Tool (Model 1)}

In the previous section it was shown that the vortex strength, as a function of velocity ratio, exhibits a selfsimilar property for various different ground clearances and approaching boundary layer combinations. This correlation is very useful from a modelling point of view, as with knowledge of the normalization parameters (i.e. $U_{\max }^{*}$ and $\Gamma_{\max }^{*}$ ), the vortex circulation, $\Gamma^{*}$, can be determined for headwind configurations. In this section an empirical vortex strength prediction tool is presented which uses the aerodynamic self-similarity property presented above, along with additional correlations to determine the vortex strength for a variety of configurations. This technique can be used to estimate the vortex strength, $\Gamma^{*}$, under headwind conditions for a specified height-to-diameter ratio, $h / D_{l}$, approaching boundary layer, $\delta^{*} / D_{l}$ and velocity ratio based on the average velocity within the sucked streamtube upstream of the intake denoted by $U^{* *},\left(=U_{i} / \bar{U}_{\infty}\right)$. The reasoning for using this velocity ratio definition rather than the standard definition based on the free-stream velocity, $U_{\infty}$, which was used in the above experiments, will be discussed below. An extension to this model is also presented in [9], which enables the corresponding distortion coefficient and vortex characteristics to be determined for any yaw angle between 0 and $90^{\circ}$.

$$
\begin{gathered}
\hat{U}=\frac{\frac{U_{\max }^{* *}}{U_{R}^{*}}-U_{R}^{*}}{1-U_{R}^{*}} \\
U_{R}^{*}=\frac{U_{c r i t}^{*}}{U_{\max }^{*}}=0.65
\end{gathered}
$$

To use the self-similar property, as shown in Fig. 5, first the trend needs to be approximated using an analytical function. To simplify this process and to enable a continuous function to be implemented, first the abscissa in Fig. 5 is transformed using Eq. (5) so that the data passes through the origin. The parameter, $U_{R}^{*}$, in Eq. (5), is equal to $U_{c r i d}^{*} / U^{*}{ }_{\max }$ which is the ratio of the vortex blow-away (or critical) velocity ratio to the velocity ratio at which the maximum strength vortex occurs, for a given non-dimensional height, as given in Eq. (6). This ratio is determined from the intersection of the linear region of the self-similar trend with the $x$-axis and is a constant equal to 0.65 (Fig. 5). This value represents the average for all configurations tested. The critical velocity ratio, $U_{c r i t}^{*}$, is the velocity ratio at which the sucked streamtube lifts off the ground plane. It has been shown from the experiments described above that $U_{c r i t}^{*}$ can be estimated using Eq. (3) when there is no 
significant approaching boundary layer thickness. However, in reality an approaching boundary layer will be present which will be an order of magnitude larger than the intake size. To account for the boundary layer thickness, $\delta$, in the model, the free-stream velocity, $\mathrm{U}_{\infty}$, in Eq. (3) should be replaced with the average velocity within the sucked streamtube, $\bar{U}_{\infty}$, as indicated in Eq. (7). Therefore to be consistent $U^{* *}$ is used in Eq. (5) which is the velocity ratio based on $\bar{U}_{\infty}$. Although the experiments described above define the velocity ratio based on the free-stream velocity, due to the self-similarity property shown in Fig. 5, it is valid to use $U^{* *}$ as the results will scale accordingly.

$U_{c r i t}^{*}=\left(\frac{U_{i}}{\bar{U}_{\infty}}\right)_{c r i t}=4 \rho^{*}\left(\frac{D_{l}}{D_{i}} \frac{h}{D_{l}}+\frac{D_{l}}{2 D_{i}}\right)$

With the self-similar profile in the modified form as shown in Fig. 8 the following empirical formula is proposed to model the self-similar profile:

$$
\frac{\Gamma^{*}}{\Gamma_{\max }^{*}}=\frac{2^{1 / k} \hat{U}}{\left[1+\hat{U}^{2 k}\right]^{1 / k}}
$$

In Eq. (8) $k$ is a constant and was determined to be 1.1 using a least squares fit of the experimental data. The model fit is shown in Fig. 8 and is plotted against the empirical data.

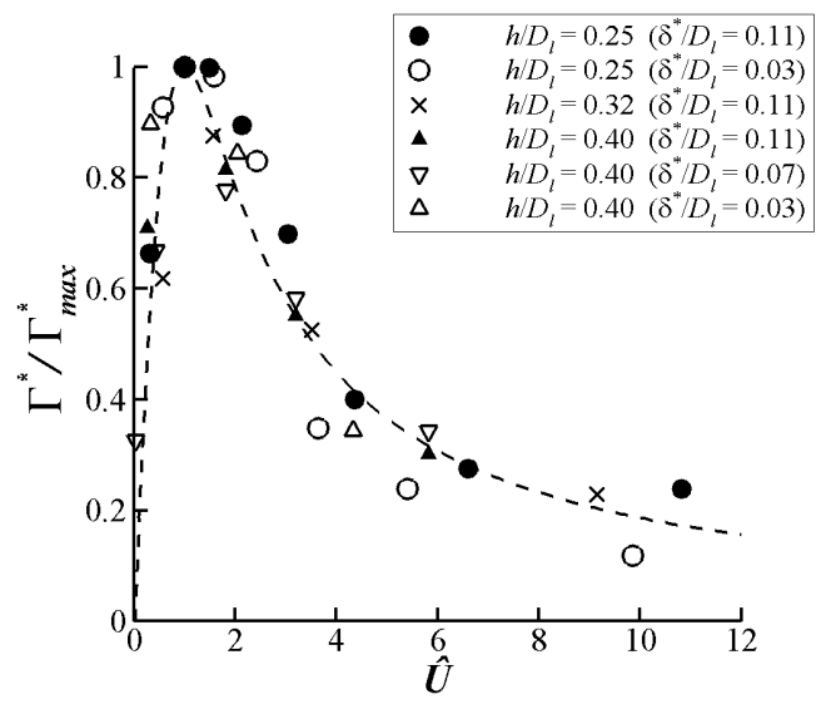

Fig. 8: The self-similar profiles of normalized vortex strength against velocity ratio with the data transformed so that the data goes through the origin.

To determine the vortex strength for a specified velocity ratio and height-to-diameter ratio $\left(h / D_{l}\right)$ knowledge of $\Gamma^{*}{ }_{\max }$ and $U_{\max }^{*}$ is required. As mentioned previously, it has been shown from the experiments that the vortex blow-away condition, $U_{\text {crit }}^{*}$, approximately matches the ideal velocity ratio when the sucked streamtube lifts off the ground plane. This criterion is given by Eq. (7) and is crucial to the model, as it accounts for the effect of ground clearance $\left(h / D_{l}\right)$ as well as the approaching boundary layer thickness. Therefore using Eq. (6) and (7), $U^{*}{ }_{\max }$ can be determined for any $h / D_{l}$. In terms of $\Gamma^{*}{ }_{\max }$, by correlating all maximum strength vortices against the corresponding velocity ratio, $U_{\max }^{*}$, (Fig. 9) a relationship has been found for all configurations studied. This is approximated by the analytical expression given in Eq. (9), using a least squares 
approach, in which the parameters $\alpha$ and $\beta$ are equal to 0.83 and -0.7 respectively. At this point it should be emphasized that use of Eq. (8) is limited to the velocity ratios below approximately 19 . This is because as the velocity ratio goes to infinity, $\Gamma^{*} / \Gamma_{\max }^{*}$, would tend to zero (i.e. the vortex strength would be zero). This of course is not the case as under quiescent conditions the vortex strength is non-zero. However, the results indicate that there is no notable difference in the strength between $U^{*}=19$ and $\infty$ (Fig. 3). Hence, it is reasonable to assume that for velocity ratios greater than 19 the flow is purely dominated by the suction induced aerodynamics and that the vortex strength is approximately equal to the no-wind vortex strength.

$$
\Gamma_{\max }^{*}=\alpha \cdot\left(U_{\max }^{*}\right)^{\beta}
$$

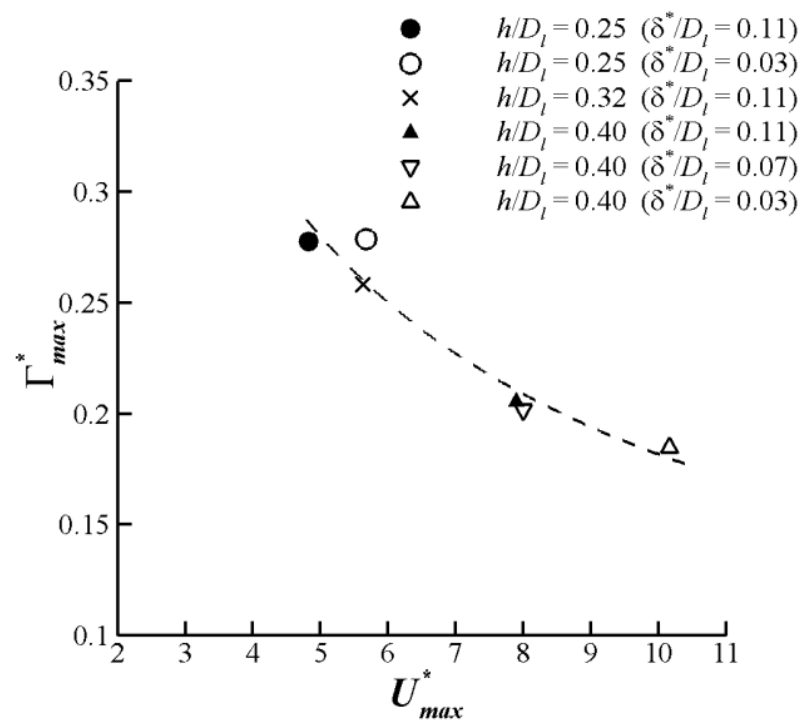

Fig. 9: Maximum vortex strength, $\Gamma_{\max }^{*}$, against the corresponding velocity ratio, $U^{*}{ }_{\max }$, for various configurations

Therefore to determine the vortex strength using the above method one must have knowledge of the intake nondimensional height, $h / D_{l}$, the average intake velocity, $U_{i}$, and also the average velocity within the sucked streamtube far upstream of the intake, $\bar{U}_{\infty}$. The first step in calculating the vortex strength for this given set of conditions is to first determine $\mathrm{U}_{\text {crit }}^{*}$ for a given value of $\mathrm{h} / \mathrm{D}_{1}$ using Eq. (7). This value is then used to determine the velocity ratio of maximum strength, $U^{*}{ }_{\max }$, using the empirical relationship given in Eq. (6). The maximum vortex strength, $\Gamma_{\max }^{*}$, can then be estimated using Eq. (9). The next step is to determine the velocity ratio, $U^{* *}$, which is the ratio $\mathrm{U}_{\mathrm{i}} / \bar{U}_{\infty}$. The value of $U^{* * *}$ along with the $U^{*}{ }_{\max }$ can then be used to determine $\hat{U}$ via Eq. (5) which therefore allows the vortex strength to be determined using the analytical expression for the self-similar profile (Eq. 8).

The method described above is one approach in estimating the vortex strength for a given headwind configuration. In some applications, such as setting boundary conditions for idealised CFD simulations, it is necessary to have knowledge of the vortex flow field as well as the overall strength. One approach is to combine the presented prediction tool with an established vortex model such as that proposed by Vatistas [21] (Eq. (10)). However to use this model, one must have knowledge of the vortex strength as well as knowledge of the vortex core radius size, $r_{c}$, and the model exponent, $n$, of the vortex.

$$
V_{\theta}=\frac{\Gamma}{2 \pi r_{c}}\left[\frac{r^{*}}{\left(1+r^{* 2 n}\right)^{1 / n}}\right]
$$


Previous research conducted by the authors [8] has shown that the individual vortex core radius, $r_{c}$, is found to be typically $3 \%$ of the intake inner diameter, $D_{i}$ [8]. In addition it has also been found that the Vatistas vortex model exponent, $n$, is generally constant across all configurations and is on average equal to 1.0 [8]. This is a significant finding as with knowledge of the vortex circulation, using the above method, the in-plane, vortex only, velocity field can be calculated for headwind configurations using the Vatistas vortex model.

\section{Analytical Vortex Strength Model}

The vortex strength prediction tool presented in the previous section is a method based solely on empirical correlations. It can be used to establish the vortex strength under headwind conditions $\left(\psi=0^{\circ}\right)$ for a non-dimensional height, approaching boundary layer, intake Mach number and velocity ratio with good accuracy. However, it is also of interest to establish a method which can predict the vortex strength and variation with velocity ratio based on a simple analytical approach. In the first part of this section a purely analytical model (Model 2) is presented which assumes that all the approaching vorticity within the sucked streamtube contributes to the overall vortex strength at the PIV plane (Fig. 1d). In the subsequent section an extension to this model is presented which also accounts for the second primary vorticity source which is associated with the induced flowfield. This method (Models 3 and 3b), is a combination of a simple analytical assessment with key empirical elements used to quantify discrete parts of the model construction.

\section{A. Model 2}

In this model, the local effect of the suction induced vorticity is neglected (Eq. (11)) and it is assumed that all the approaching vorticity within the sucked streamtube contributes to the overall vortex strength at the PIV plane (Fig. 1d)

$$
\Gamma^{*}=\Gamma_{\infty}^{*}
$$

The approaching boundary layer circulation, $\Gamma_{\infty}$, is determined by integrating all the captured boundary layer vorticity within the whole cross-section of the sucked streamtube, at a far-field location upstream of the intake. The upstream sucked streamtube size is determined from mass conservation based on the conditions within the intake and the upstream reference velocity $\left(U_{\infty}\right)$ as will be discussed below. At this location it is assumed that the flow is parallel to the surface and that the only source of vorticity is due to the variation in streamwise velocity, $u$, which varies with distance from the wall, $z$. Therefore the approaching vorticity can be given by Eq. (12).

$\omega_{\infty} \approx-\frac{\partial u}{\partial z}$

Boundary layer measurements in the wind tunnel show that the velocity profile follows a power law approximation, of the form given in Eq. (13), with $n_{b l} \approx 7$ (see [9] for further details). The vorticity can therefore be determined by taking the derivative of Eq. (13) which has been evaluated using a second order central difference scheme with a forward difference being implemented at the wall.

$$
\frac{u(z)}{U_{\infty}}=\left(\frac{z}{\delta}\right)^{\frac{1}{n_{b l}}}
$$

To integrate the vorticity distribution within the capture streamtube an estimation of the shape and length scale of the sucked streamtube size, $L_{q}$, is required (Fig. 10a). In this study the undisturbed sucked streamtube is assumed to be initially circular and $L_{q}$ is defined using Eq. (14). The radius of the sucked streamtube, $r_{\infty}$, is determined from the continuity equation and therefore Eq. (14) can be rearranged as shown in Eq. (15)-(17). However due to the close proximity of the ground there will be a proportion of the total area, $A_{\text {ug }}$ 
that will be below the ground plane (Fig. 10a). This, of course, is not plausible and conservation of mass states that this area must be accounted for elsewhere. In this study it is assumed that the nominal sucked streamtube remains circular when interacting with the ground with its radius increasing by an amount, $\Delta r$, such that the area above the ground equals the required sucked streamtube area, $A_{\infty}$ (Fig. 10b). The same approach is used when accounting for the approaching boundary layer thickness. Hence when $\delta$ is increased, the velocity deficit within the boundary layer will increase and therefore the streamtube size will have to increase for the mass flow to remain constant. In this case again the sucked streamtube stays circular with its radius increasing by the amount required to satisfy mass conservation.

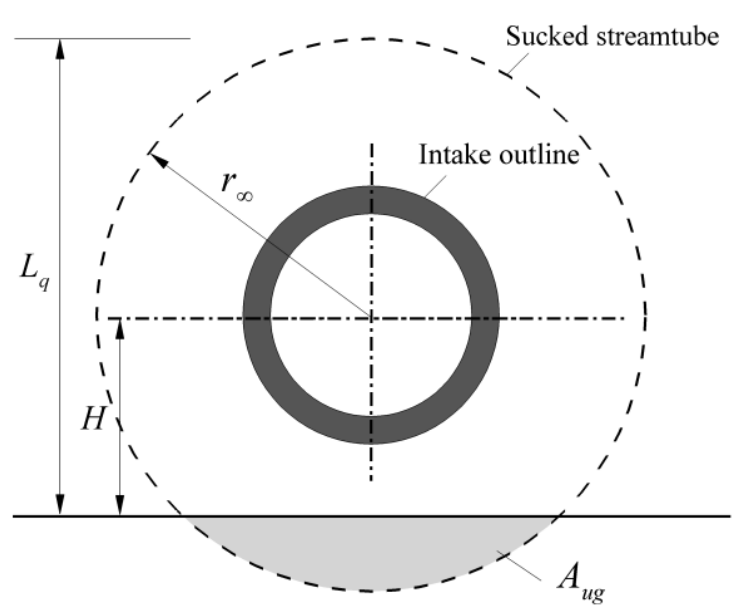

(a)

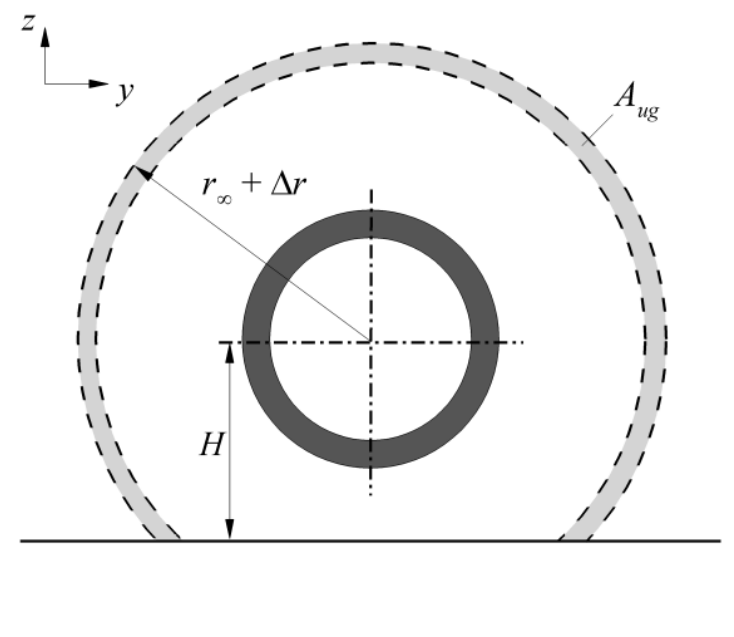

(b)

Fig. 10: The effect of ground interaction on the sucked streamtube characteristics where (a) is the nominal undisturbed sucked streamtube and (b) is the altered capture streamtube after ground interaction.

$L_{q}=H+r_{\infty}$

$L_{q}=H+\sqrt{\frac{1}{\pi}\left(\frac{\dot{m}}{\rho_{\infty} \bar{U}_{\infty}}\right)}$

where

$\bar{U}_{\infty}=\frac{1}{A_{\infty}} \int_{A_{\infty}} u(z) d A_{\infty}$

and

$$
A_{\infty}=\frac{\dot{m}}{\rho_{\infty} \bar{U}_{\infty}}
$$

As mentioned above, a vortex can only form if $r_{\infty}>H$. Since $\bar{U}_{\infty}$ depends on $A_{\infty}$ and vice versa, a simple iterative scheme is required to determine, $r_{\infty}$ and therefore $L_{q}$. From this estimate of the sucked streamtube size and shape, the total circulation within the capture streamtube, $\Gamma_{\infty}$, can be calculated using Eq. (18). The 
integration was performed using a numerical trapezoidal scheme for half the sucked streamtube in the positive $y$-direction and symmetry was applied to determine the total circulation.

$\Gamma_{\infty}=\int_{A_{\infty}} \omega_{\infty} d A_{\infty}$

In summary, to use this method the boundary layer characteristics are required which includes the thickness, $\delta$, the shape factor, $n_{b l}$, and also a reference velocity. These parameters enable the boundary layer profile to be established which in turn allows the approaching vorticity to be quantified. In addition the intake ground clearance, $h / D_{l}$, and mass flow rate, $m$ are necessary elements which along with the velocity profile enables the sucked streamtube size and level of interaction with the ground plane to be determined. The approaching vorticity can then be integrated within the sucked streamtube which provides an estimate for the ground vortex strength. In the following section the results of the model are presented and compared to the experimental dataset.

\section{Results and Discussion}

The total vorticity within the sucked streamtube at the far-field was integrated for the full range of investigated velocity ratios. The results for the model at a non-dimensional height, $h / D_{l}$, of 0.25 with an intake diameter, $D_{l}$ and velocity, $U_{i}$, equal to model scale (i.e. $0.12 \mathrm{~m}$ and $190 \mathrm{~m} / \mathrm{s}$ respectively) is shown in Fig. 11a. This is for a configuration with an approaching boundary layer, $\delta^{*} / D_{l}$ of 0.11 . Also shown in the figure are the experimental results under the same conditions. Although there are important differences between the experimental measurements and the Model A predictions, there are some key aspects that the model captures. In particular, it captures the rapid rise in vortex strength as the velocity ratio increases from the critical ratio $\left(U_{c r i t}^{*}\right)$ followed by a local maximum. It gives reasonable agreement on the value of peak circulation which is within $8 \%$ of the experiments. Furthermore, the corresponding velocity ratio $\left(U_{\max }^{*}\right)$ is predicted to be 6.33 which compares to 4.9 for the experiments (Fig. 11a). However, the key difference is for the higher velocity ratios where the model fails to capture the reduction in strength and at a $U^{*}$ of 19 the vortex strength is over predicted, by a factor of three times. Nevertheless, the model demonstrates some useful characteristics for such a simple method.

(a)

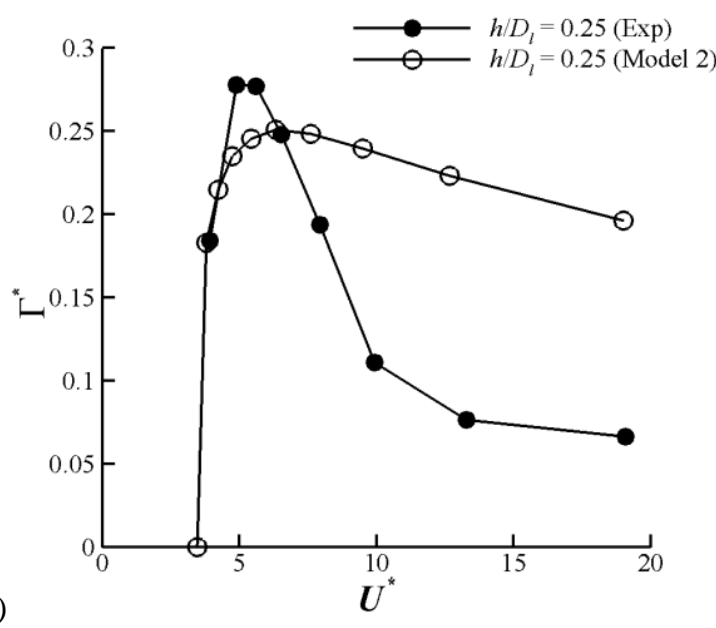

(b)

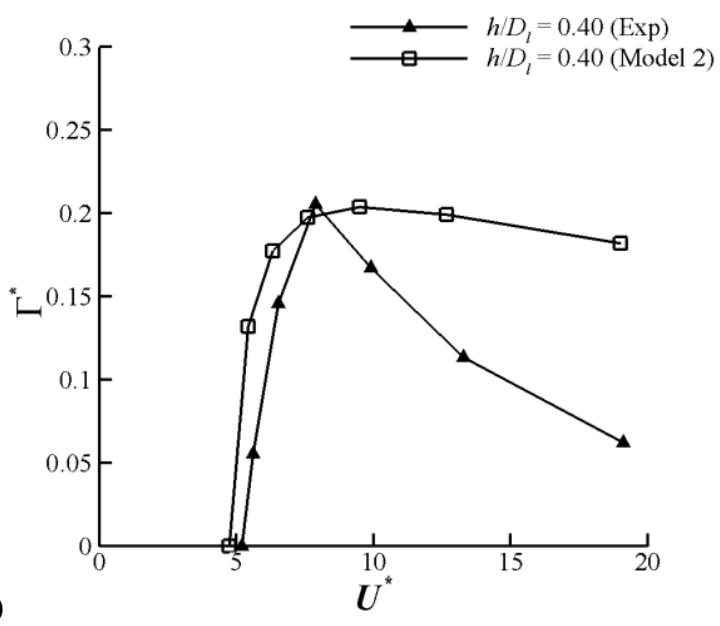

Fig. 11: The total non-dimensional vortex strength against velocity ratio for Model $A$ with comparison to the experiments for (a) $h / D_{l}=0.25$ and (b) $h / D_{l}=0.40 .\left(\delta^{*} / D_{l}=0.11, M_{i}=0.58\right)$.

Predictions have also been computed for the highest ground clearance of 0.4 for direct comparison with the experimental results as shown in Fig. 11b. In general, as the ground clearance increases the peak strength prediction improves, where at $h / D_{l}=0.4$, the value is within $0.8 \%$ of the experiments. Again the corresponding 
velocity ratio has been slightly over predicted at $9.52\left(U_{\max }^{*}\right)$ in comparison to 7.88 for the experiments. As with the results for the lower height-to-diameter ratio, there is little change in the predicted strength between these two velocity ratios. The relative difference between the model and experimental results at high velocity ratios is roughly the same for all investigated non-dimensional heights and is over predicted by three times approximately. This perhaps demonstrates that the discrepancy at high velocity ratios is not a function of ground clearance, but rather there is an additional mechanism that is not being captured by the model. This additional mechanism is clearly expected to be associated with the influence of the induced vorticity source, which is expected to be strongly influential at high velocity ratios.

\section{B. Model 3}

As mentioned in the previous section the poor agreement between Model 2 and the experiments at high velocity ratios indicates that there is an additional feature that is not being captured by the model. One limitation of the analytical approach employed by Model 2 is that the induced circulation magnitude cannot be determined explicitly and is therefore not taken into account. The subsequent developments of Model 3 and $3 b$ use some of the experimental results to provide empirical data which is used to address the elements of the model which cannot be determined analytically. Given the rolling road results presented in Fig. 7, the induced circulation is a significant aspect and is almost certainly why there is such a poor prediction at high velocity ratios. As explained above, the moving ground technique allows the variation in the induced circulation, $\Gamma_{i d}$, with velocity ratio to be estimated. The results can therefore be used within a prediction model so that both the approaching and induced circulation sources are taken into account.

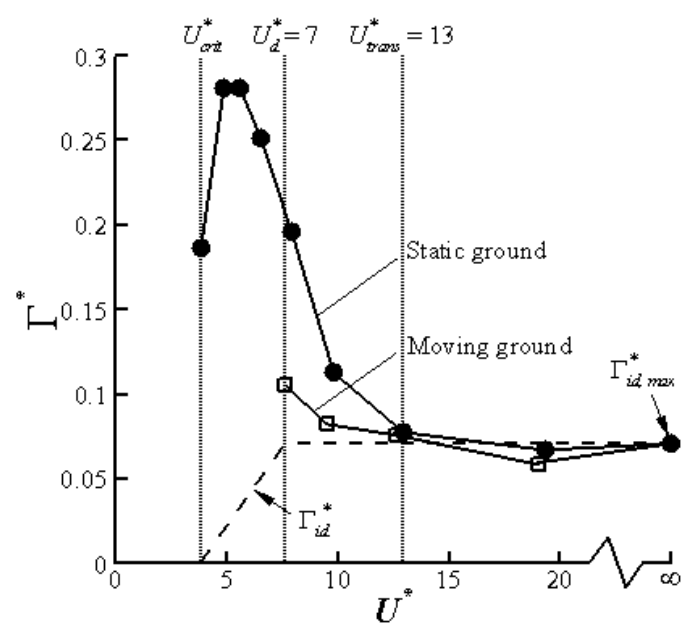

Fig. 12: Vortex strength against velocity ratio for static and moving ground cases illustrating the approximation used for the induced circulation, $\Gamma_{i d}^{*}$, variation with velocity ratio in Model 3.

The vortex strength measurements for the rolling road configuration are again shown in Fig. 12 which includes the static ground plane results in an increasing headwind. Within the figure, two new variables are introduced denoted by $U_{\text {trans }}^{*}$ and $U_{d}^{*}$. It is evident from the moving ground results that when the velocity ratio exceeds a certain limit the approaching vorticity has no influence on the overall vortex strength. This limit is denoted by $U_{\text {trans }}^{*}$ in Fig. 12 and is approximately equal to 13 for a non-dimensional height of $0.25\left(h / D_{l}\right)$. In addition, for the velocity ratios investigated (i.e. $\left.7<U^{*}<\infty\right)$ for this ground clearance $\left(h / D_{l}=0.25\right)$, the vortex strength is approximately constant and its magnitude is roughly equal to the quiescent configuration $\left(U^{*}=\infty\right)$. As no further measurements were taken at lower velocity ratios there is no indication of what happens to the vortex strength as the velocity ratio reduces further towards the critical condition $\left(U_{c r i t}^{*}\right)$. Since by definition the induced circulation is created due to the interaction of the sucked streamtube with the ground, when the streamtube lifts off the surface, $\Gamma^{*}{ }_{i d}$ must be reduced to zero. Hence with no further data available it is assumed that there is a simple linear reduction in the vortex strength from $U^{*}=7$ (denoted by $U_{d}^{*}$ in Fig. 12), where the 
vortex strength is approximately equal to the quiescent configuration, to the blow-away condition, $U_{c r i t}^{*}$, where the vortex strength is zero (Fig. 12). This is expressed in Eq. (19) and is also been plotted in Fig. 12 (Note that Eq. (19) applies only to an $h / D_{l}=0.25$ ).

$\Gamma_{i d}^{*}=\left\{\begin{array}{cc}\frac{\left(U^{*}-U_{c r i t}^{*}\right)}{\left(7-U_{c r i t}^{*}\right)} \Gamma_{i d, \text { max }}^{*} & U^{*}<7 \\ \Gamma_{i d, \text { max }}^{*} & U^{*}>7\end{array}\right.$

Where $\Gamma^{*}{ }_{i d, \max }$ is determined from Eq. (20) which is taken from a least squares curve fit of the experimental data presented in Fig. 2.

$\Gamma_{i d, \max }^{*}=0.0125 \cdot\left(\frac{h}{D_{l}}\right)^{-1.2}$

Now that a prediction of both the approaching, $\Gamma_{\infty}$, and induced circulation, $\Gamma_{i d}$, sources is available both must now be combined to give the overall vortex strength, $\Gamma^{*}$. Given the rolling road experimental observations it is assumed that, when the velocity ratio is greater than $U_{\text {trans }}^{*}$, only the induced circulation source contributes to the overall vortex strength (Eq. (21)). However, when the velocity ratio reduces below $U_{\text {trans }}^{*}$ the overall vortex strength is given by a linear difference of the two circulation sources (Eq. (21)). This is because the two sources have opposite orientations (Fig. 1b \& d).

$\Gamma^{*}=\left\{\begin{array}{cl}\Gamma_{\infty}^{*}-\Gamma_{i d}^{*} & U^{*}<U_{\text {trans }}^{*} \\ \Gamma_{i d}^{*} & U^{*}>U_{\text {trans }}^{*}\end{array}\right.$

However, to determine the induced circulation variation for various non-dimensional heights the variation of transition velocity ratio, $U_{\text {trans }}^{*}$, and the induced circulation reduction velocity ratio, $U_{d}^{*}$ with ground clearance must be determined. Given that the vortex strength against velocity ratio trends exhibit a self-similar property (Fig. 5) it follows that both $U_{\text {trans }}^{*}$ and $U_{d}^{*}$ should scale with the critical velocity ratio, $\mathrm{U}_{\text {crit }}^{*}$ Therefore since $U^{*}$ trans and $U_{d}^{*}$ are equal to 13 and 7 respectively for a non-dimensional height of $0.25\left(h / D_{l}\right)$ (Fig. 12) and $U_{c r i t}^{*}$ is equal to 3.88 for this ground clearance (Eq. (3)), two new empirical constants can therefore be constructed which enable $U_{\text {trans }}^{*}$ and $U_{d}^{*}$ to be determined for any non-dimensional height. These empirical constants are denoted by $\Pi_{1}$ and $\Pi_{2}$ as shown in Eq. (22) and Eq. (23) respectively.

$$
\begin{aligned}
& \Pi_{1}=\frac{U_{\text {trans }}^{*}}{U_{c r i t}^{*}}=3.4 \\
& \Pi_{2}=\frac{U_{d}^{*}}{U_{c r i t}^{*}}=1.8
\end{aligned}
$$

The induced circulation variation can therefore be given by Eq. (24) and the total vortex strength can now be calculated using Eq. (21) for any non-dimensional height under pure headwind conditions.

$$
\Gamma_{i d}^{*}=\left\{\begin{array}{cl}
\frac{\left(U^{*}-U_{c r i t}^{*}\right)}{\left(U_{d}^{*}-U_{c r i t}^{*}\right)} \Gamma_{i d, \text { max }}^{*} & U^{*}<U_{d}^{*} \\
\Gamma_{i d, \text { max }}^{*} & U^{*}>U_{d}^{*}
\end{array}\right.
$$


To apply Model 3 to a particular configuration firstly the approaching circulation should be calculated as outlined above for Model 2. The next task is to calculate the induced circulation variation with velocity ratio which is determined purely from empirical correlations. This is performed by firstly computing the maximum induced circulation, $\Gamma_{i d, \max }^{*}$, using Eq. (20) based on the non-dimensional height in question. $\mathrm{U}^{*}{ }_{\mathrm{d}}$ must then be established using Eq. (23) which then allows the variation in the induced circulation across the full range of velocity ratios to be estimated using Eq. (24). Then the velocity ratio at which the approaching vorticity becomes influential on the total vortex strength should be calculated using the above empirical relationship (Eq. (22)). Finally the induced and approaching circulation sources should be combined to provide an estimate of the overall vortex strength using Eq. (21).

The reader should bear in mind that the empirical constants are derived purely from the $h / D_{l}=0.25$ dataset. The uncertainty of the values is determined from the resolution of the acquired data. The transition velocity ratio, $U_{\text {trans }}^{*}$, is defined as being the velocity ratio at which the static ground plane and rolling road results diverge (Fig. 12). Based on the results, $U_{\text {trans }}^{*}$ will have a positive bias uncertainty, since the transition could occur at a lower but not at a higher velocity ratio. For a similar reason this is also the case for $U_{d}^{*}$. The overall uncertainty is therefore dominated by the resolution of the experimental data points and is estimated to be approximately +0.4 and +0.2 for $\Pi_{1}$ and $\Pi_{2}$ respectively.

\section{Results and Discussion}

The results for Model 3 are shown in Fig. 13 for the datum configuration $\left(h / D_{l}=0.25, \delta^{*} / D_{l}=0.11\right)$ and are compared with Model 2 and the experiments. The variation of vortex strength with velocity ratio now shows good agreement with the experimental results and is significantly improved relative to the Model 2 predictions. In particular, the velocity ratio for the peak vortex strength, $U_{\max }^{*}$, is correctly captured along with the subsequent decay with further increases in velocity ratio. Clearly the value of $\Gamma^{*}$ under quiescent conditions $\left(U^{*}=\propto\right)$ is locked into the model through the empirical correlation presented in Fig 2 which captures the maximum induced vorticity, $\Gamma_{i d}^{*}$. However the vortex strength at lower velocity ratios $\left(U^{*}<12\right)$, where both the induced and approaching circulation sources contribute, is under predicted (Fig. 13).

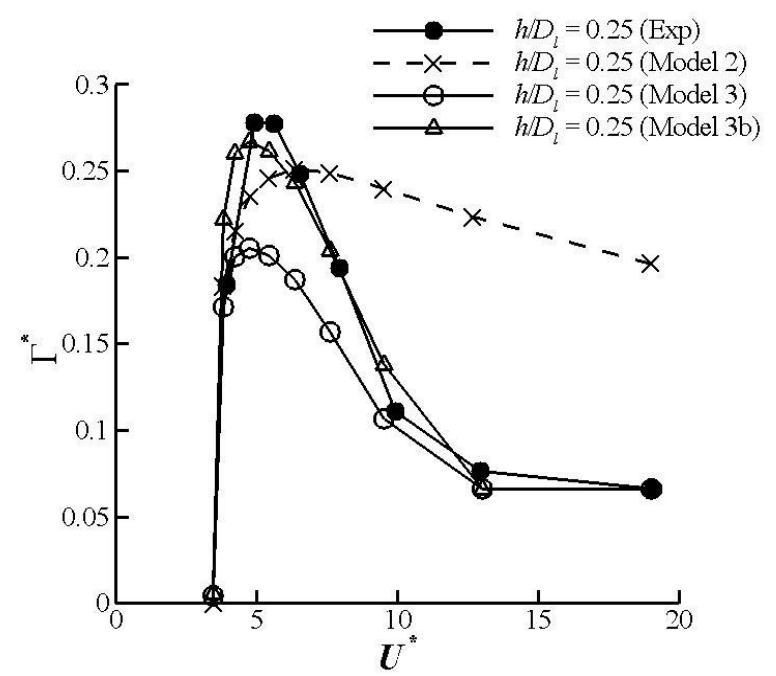

Fig. 13: Predicted non-dimensional vortex strength against velocity ratio for Model 3 with comparison to Model 2 and the experimental results $\left(h / D_{l}=0.25, \delta^{*} / D_{l}=0.11, M_{i}=0.58\right)$

To match the predicted peak vortex strength, $\Gamma_{\max }^{*}$, with the experimental peak circulation a final empirical constant, $\sigma$, has been applied (Eq. (25)). The empirical constant is expected to represent the inaccuracies in the prediction of the induced circulation, the interaction between the two primary vorticity sources and also the assumptions relating the sucked streamtube size, shape and interaction with the ground plane. The empirical constant, $\sigma$, has been determined for all three ground clearance configurations and was found to be 1.35 for both an $h / D_{l}=0.25$ and 0.32 but reduced to 1.18 for an $h / D_{l}=0.4$. This decrease in $\sigma$ was 
anticipated and is expected to be a result of the reduced interaction of the sucked streamtube with the ground and also a smaller interaction between the two primary vorticity sources.

$$
\sigma \cdot\left(\Gamma_{\max }^{*}\right)_{\bmod e l}=\left(\Gamma_{\text {max }}^{*}\right)_{\exp }
$$

Based on the above findings the average value of $\sigma$ is applied to all investigated non-dimensional heights and is equal to 1.3. Since this empirical factor primarily represents the interaction between the induced and approaching vorticity sources, $\sigma_{A V}$ is only enforced for velocity ratios in which both vorticity sources contribute to the overall vortex strength (Eq. (26)).

$\Gamma^{*}=\left\{\begin{array}{cc}\sigma_{a v}\left(\Gamma_{\infty}^{*}-\Gamma_{i d}^{*}\right) & U^{*}<U_{\text {trans }}^{*} \\ \Gamma_{i d}^{*} & U^{*}>U_{\text {trans }}^{*}\end{array}\right.$

With the empirical constant applied the modified model is now identified as $3 \mathrm{~b}$ and the predicted vortex strength with velocity ratio is plotted in Fig. 13 and compared with Model 3 for the datum intake ground clearance of $0.25\left(h / D_{l}\right)$. The prediction now shows excellent agreement with the experimental data. Model $3 \mathrm{~b}$ is also tested for the other configurations $\left(h / D_{l}=0.32,0.40\right)$ as shown in Fig. 14. The results for the model at a non-dimensional height, $h / D_{l}$, of 0.32 (Fig. 14a) again shows good agreement with the experimental results. This perhaps underwrites the modelling assumptions relating to the induced circulation and velocity ratio at which the approaching circulation becomes influential. At the higher height-to-diameter ratio $\left(h / D_{l}\right)$ of 0.4 the comparison with the experimental data is reasonable although the vortex strength is slightly over predicted (Fig. $14 \mathrm{~b})$. This is anticipated as the average value of the empirical constant across all non-dimensional heights is larger than the value specific to this height $\left(h / D_{l}=0.4\right)$.
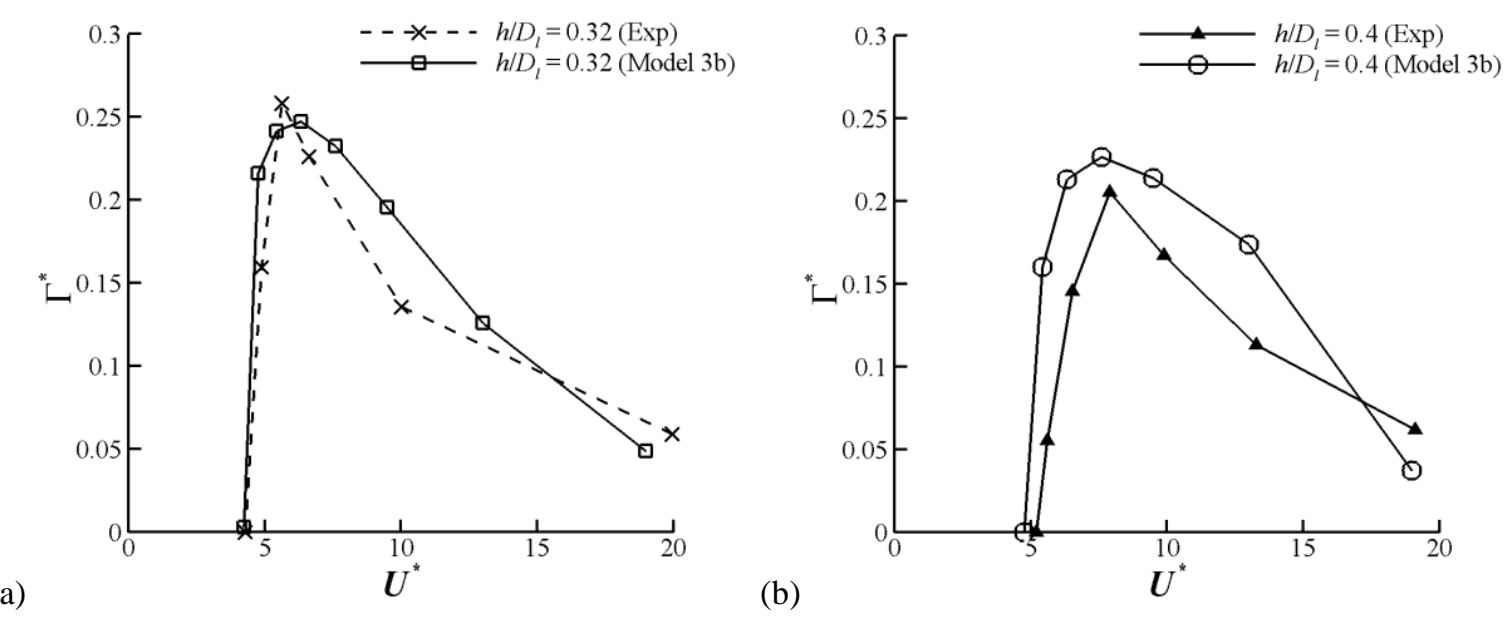

Fig. 14: Predicted total non-dimensional vortex strength against velocity ratio with the empirical constant applied for (a) $h / D_{l}=0.32$, and (b) $h / D_{l}=0.40$ with $\delta / D_{l}=0.11, M_{i}=0.58$

\section{Conclusions}

Three prediction techniques have been proposed and developed which estimate the ground vortex strength under headwind conditions. The first tool employs a purely empirical approach which uses a number of experimentally derived correlations. The second technique employs a purely analytical approach in which only the approaching vorticity source is considered. Whereas the third model, which is a more advanced version of the second model, uses both analytical and empirical elements. For this model both of the primary circulation 
sources are considered which are associated with the approaching and induced flowfields, the latter of which has been determined empirically.

The empirical model provides the most accurate representation of the vortex characteristics for a given configuration. It can be used to determine the vortex strength for different non-dimensional heights, approaching boundary layer, intake Mach number, and velocity ratio. Most significantly, it identifies the blow-away condition as well as the operating point that generates the strongest vortex and is a simple tool for preliminary design assessments. This is useful in identifying the important operating points for further investigation using full scale engine tests or CFD simulations.

The second and third models are less accurate but are more instructive in further understanding the underlying mechanisms of headwind ground vortex formation. The second model can be used to give an approximate estimation of the peak vortex strength for a given intake non-dimensional height and approaching boundary layer without any need for empirical data, however it fails to identify the correct velocity ratio at which this condition occurs. In addition the model over predicts the vortex strength at high velocity ratios which highlights the significant influence of the induced vorticity source at these conditions. By accounting for the suction induced vorticity source a combined analytical and empirical tool is developed which provides good agreement with the experimental results in capturing the key ground vortex characteristics.

\section{Acknowledgements}

J. Murphy was funded by an EPSRC studentship. The authors would like Rolls-Royce for their technical and financial support. Particular thanks go to Chris Sheaf of Installations and Controls Engineering and Jeff Green of Fan Systems for their valued input in this research.

\section{References}

[1] Rodert, L.A., and Garrett, F.B., "Ingestion of Foreign Objects into Turbine Engines by Vortices," National Advisory Committee for Aeronautics, NACA TN 3330, 1953.

[2] Glenny, D.E., "Ingestion of Debris into Intakes by Vortex Action," Ministry of Technology, Aeronautical Research Council, C.P. No. 1114, 1968.

[3] Motycka, D.L., "Ground Vortex - Limit to Engine/Reverser Operation," American Society of Mechanical Engineers, Paper 75-GT-3, 1975.

[4] Green, J.S., "Forced Response of a Large Civil Fan Assembly," American Society of Mechanical Engineers, Paper GT2008-50319, June 2008.

[5] de Siervi, F., Viguier, H.C., Greitzer, E.M., "Mechanisms of Inlet Vortex Formation," Journal of Fluid Mechanics, Vol. 124, 1982, pp. 173-207. doi: $10.1017 / \mathrm{S} 0022112082002456$

[6] Shin, H.W., Cheng, W.K., Greitzer, E.M., "Inlet Vortex Formation due to Ambient Vorticity Intensification," AIAA Journal, Vol. 24, No. 4, 1986, pp. 687-689. doi: $10.2514 / 3.9330$

[7] Murphy, J. P. and MacManus, D. G., "Ground Vortex Aerodynamics under Crosswind Conditions," Experiments in Fluids, published online 10 Jun. 2010 doi: 10.1007/s00348-010-0902-4

[8] Murphy, J. P. and MacManus, D. G., "Intake Ground Vortex Aerodynamics under Headwind Conditions," Journal of Aerospace Science and Technology (Under review).

[9] Murphy, J.P., "Intake Ground Vortex Aerodynamics," PhD Thesis, Cranfield University, 2009.

[10] Murphy, J., MacManus, D. and Sheaf, C., "An Experimental Investigation into the Formation of Ground Vortices during Take-off," AIAA Journal, Vol. 48 no. 3, March 2010 doi: $10.2514 / 1.45896$

[11] Jeong, J., and Hussain, F., "On the Identification of a Vortex," Journal of Fluid Mechanics, Vol. 285, 1995, pp. 69-94. doi: $10.1017 /$ S0022112095000462

[12] Burley, C.L., Brooks, T.F., van der Wall, B., "Rotor Wake Vortex Definition - Initial Evaluation of 3-C PIV Results of the Hart-II Study," number 50, $28^{\text {th }}$ European Rotorcraft Forum, Bristol, England, 17-20 September 2002. 
[13] Taylor, J.R., "An Introduction to Error Analysis: The Study of Uncertainties in Physical Measurements," University Science Books, California, 1982.

[14] Raffel, M., Willert, C., and Kompenhans, J., Particle Image Velocimetry: A Practical Guide, SpringerVerlag, Berlin, 1998.

[15] Petracci, A., van Doorne, C. W. H., Westerweel, J., "Analysis of Stereoscopic PIV Measurements using Synthetic Images," Particle Image Velocimetry: Recent Improvements, Springer, Berlin, 2004.

[16] Prasad, A.K., "Stereoscopic Particle Image Velocimetry," Experiments in Fluids, Vol. 29, No. 2, 2000, pp. 103-116. doi: $10.1007 / \mathrm{s} 003480000143$

[17] Brix, S., Neuwerth, G., and Jacob, D., "The Inlet-Vortex System of Jet Engines Operating near the Ground", AIAA paper 2000-3998, 2000.

[18] Nakayama, A. and Jones, J. "Vortex Formation in Inlet Flow near a Wall”, AIAA Paper 96-0803, 1996.

[19] Zantopp, S. "Jet Engine Ground Vortex Studies" MSc. Thesis, Cranfield University, 2008

[20] Zantopp, S., MacManus, D., and Murphy, J., "Computational and Experimental Study of Intake Ground Vortices", Royal Aeronautical Journal, in press.

[21] Vatistas, G. H., Kozel, V., and Mih, W. C. "A Simpler Model for Concentrated Vortices", Experiments in Fluids, Vol. 11, 1991, pp. 73-76. 


\section{Cranfield University}

\section{CERES https://dspace.lib.cranfield.ac.uk}

2011-02-28

\section{Intake Ground Vortex Prediction Methods}

Murphy, J. P.

AIAA

JP Murphy and DG MacManus, Journal of Aircraft, 2011, Vol. 48, Iss. 1, pp23-33

http://dx.doi.org/10.2514/1.46221

Downloaded from Cranfield Library Services E-Repository 